\title{
Rehidratación Oral en Lactantes con Diarrea Aguda
}

\author{
Dr. Armando Caichac R. ${ }^{1}$; Dra. Carmen Luz Avilés L. ${ }^{2}$; \\ E.U. Julia Romero T. ${ }^{3}$; E.U. Gabriela Pinto C. ${ }^{4}$.
}

\section{Oral Rehydration Therapy in Infants with Acute Diarrhea}

\begin{abstract}
Oral rehydration therapy (ORT) and parenteral therapy (PT) were compared in infants with mild to moderate dehydration associated to acute diarrheal syndrome. Twenty three of the twenty seven patients on ORT were clinically well hydrated after six hours of treatment. Five of ten patients on PT were well hydrated after six hours. The difference between both goups is significant at $p<0.05$. No differences in fecal nor oral looses (vomitus) were detected between ORT success and failure patients. The successful ORT patients had greater oral intakes than those considered as failures ( 79 vs. $60 \mathrm{ml} \times \mathrm{kg}$ in first six hours). No serum sodium value differences were appreciated between $O R T$ and PT patients. Hypematremia was not detected among the study groups.
\end{abstract}

(Key words: Acute diarrhea. Dehydration. Infants. Oral therapy).

La información actualmente disponible permite establecer que la administración de una solución oral que contenga glucosa y electrolitos es efectiva y segura para el tratamiento de adultos y niños con deshidratación leve y moderada debida a diarrea aguda ${ }^{1}$.

Los expertos de la OMS han desarrollado una solución que contiene $3.5 \mathrm{~g}$ de cloruro de sodio, $1.5 \mathrm{~g}$ de cloruro de potasio, $2.5 \mathrm{~g}$ de bicarbonato de sodio y $20 \mathrm{~g}$ de glucosa por cada litro, para ser empleada en adultos y niños con cólera en áreas donde es prevalente ${ }^{2}$.

La misma solución ha sido empleada con buen éxito en lactantes y niños con diarrea no colérica, administrándola en forma alternada con agua en proporción de 2:1, para soslayar riesgos de hipernatremia iatrogénica ${ }^{3} \cdot 4-5-6-7 \cdot 8-9$.

El objetivo de este trabajo fue repetir estas experiencias en el Hospital de Rancagua y compararlas con la terapia parenteral en un grupo de lactantes hospitalizados por diarrea aguda con deshidratación.

\section{PACIENTES Y METODO}

El estudio incluyó a 27 lactantes que cumplían los siguientes requisitos: edad de uno a 24 meses, diarrea aguda de menos de cuatro días de

1. Médico Servicio Neonatología Hospital de Rancagua.

2. Médico Servicio de Pediatría Hospital de Rancagua.

3. Enfermera supervisora Servicio de Pediatría, Hospital de Rancagua.

4. Enfermera Servicio de Pediatría Hospital de Rancagua. evolución, deshidratación leve o moderada, ausencia de shock, ileo o compromiso de concien. cia.

Se definieron la deshidratación leve y moderada como aquellas con pérdida del peso corporal de menos de $5 \%$ y menos de $10 \%$ respectivamente; como natremia normal se definió el rango entre 130 y $150 \mathrm{mEq}$ por litro 10 .

Los pacientes considerados aptos para ser incorporados al estudio, fueron ingresados al servicio de pediatria y sometidos durante seis horas a controles de balance hidrico; como tratamiento se les dio a beber a voluntad solución OMS alternada con agua de cane]a, en proporción de $100 \mathrm{ml}$ de agua de canela por cada $200 \mathrm{ml}$ de solución. Se tomaron muestras para electrolitos plasmáticos al ingreso, a las seis y 24 horas de evolución, evaluándose simultáneamente su estado de hidratación.

Cumplidas seis horas de tratamiento, los pacientes que estaban bien hidratados recibieron alimentación con leche (26\% de materia grasa), preparada al $5 \%, 100 \mathrm{ml} \times \mathrm{kg} \times$ dia; comida sin residuos de acuerdo a edad y solución OMS en volunen aproximado a los $100 \mathrm{ml} \mathrm{x} \mathrm{kg} \times$ dia. En el segundó día se suministró leche al $10 \%$ y solución OMS a voluntad, para descontinuarla al tercer día, completando el volumen necesario de líquidos con leche, comidas y agua a voluntad.

Se consideró que se habia terido buen éxito con la terapia oral si los pacientes estaban bien hidratados a las seis horas de tratamiento $\mathrm{y}$ fracasos a los que aún tenían signos clínicos de deshidratación; estos últimos, recibieron tratamiento parenteral a partir del momento señalado.

El grupo control estuvo constituido por diez 
lactantes que cumplían idénticos requisitos. Estos fueron sometidos a los mismos registros y exámenes pero recibieron hidratación por vía parenteral con solución de glucosa al $5 \%$ en cantidad de $180-220 \mathrm{ml} \times \mathrm{kg} \times$ dia, sodio $10-12 \mathrm{mEq} \times \mathrm{kg} \times$ día, potasio $3-4 \mathrm{mEq} \times \mathrm{kg} \mathrm{x}$ día y bicarbonato de sodio en caso de acidosis clínica 3-4 mEq x kg x dia.

Los grupos de control y de rehidratación oral (en adelante TRO), se complementaron secuencialmente; la única diferencia encontrada entre ambos ocurrió con el sexo, pues hubo mayor proporción de hambres en el grupo de TRO (Tabla 1), diferencia que se consideró sin influencia en los resultados.

Para el análisis estadístico, se aplicaron pruebas de chi cuadrado y $T$ de Student; se consideraron estadísticamente significativos valores de $\mathrm{p}+0.05$.

Tabk I.

Características de los Pacientes al Ingtesar al Estudio.

\begin{tabular}{|c|c|c|}
\hline Características & $\begin{array}{l}\text { GRUPO } \\
\text { TRO }\end{array}$ & Control \\
\hline$N^{\circ}$ Pacientes & 27 & 10 \\
\hline Hombres: Mujeres(i) & $20: 7$ & $5: 5$ \\
\hline \multicolumn{3}{|l|}{ Edad (Meses) } \\
\hline $0-5$ & 17 & 5 \\
\hline $6-11$ & 9 & 4 \\
\hline $12-24$ & 1 & 1 \\
\hline \multicolumn{3}{|l|}{ Estado Nutricional } \\
\hline Eutrofico & 19 & 8 \\
\hline D l & 5 & 2 \\
\hline D II & 3 & 0 \\
\hline \multirow{2}{*}{\multicolumn{3}{|c|}{$\begin{array}{l}\text { Grado de } \\
\text { Deshidratación(x) }\end{array}$}} \\
\hline & & \\
\hline Leve & 22 & 9 \\
\hline Moderado & 5 & 1 \\
\hline
\end{tabular}

(i): Diferencia estadísticamente significativa para $\mathrm{p}<0.05$

(x): Calculado en base al aumento de peso al hidratatse.

\section{RESULTADOS}

Después de seis horas con TRO, los signos de deshidratación desaparecieron completamente en 23/27 pacientes; de los cuatro restantes, tres recibieron terapia parenteral y otro continuó con TRO, hidratándose en 24 horas. En el grupo control sólo $5 / 10$ pacientes estaban bien hidratados al cabo de seis horas; la diferencia con el gripo anterior es significativa (Tabla 2); a las 24 horas, 9/10 pacientes de este grupo estaban bjen hidratados.
Tabla 2.

Estado de Hidratación a las seis horas de Tratamiento en los Grupos con Terapia Ora! (TRO) y con Terapia Parenteral (Control)

\begin{tabular}{lccc}
\hline & $\begin{array}{c}\text { Bien } \\
\text { Hidratado }\end{array}$ & Deshidratado & Total \\
\hline TRO & $23(\mathrm{x})$ & 4 & 27 \\
Control & $5(\mathrm{x})$ & 5 & 10 \\
Total & 28 & 9 & 37 \\
\hline
\end{tabular}

(x): Diferencia estadísticamente significativa para $\mathrm{p}<0.05$.

Sólo 6/27 pacientes tratados por vía oral tuvieron vómitos, las pérdidas por esta vía no influyeron en el resultado del tratamiento.

Las pérdidas por deposiciones fueron un promedio $3.3 \mathrm{ml} \mathrm{x} \mathrm{kg} \mathrm{x}$ hr., no encontrándose tampoco diferencias entre los pacientes hidratados y los fracasos de la TRO.

Las pérdidas por deposiciones del grupo control, fueron $1.6 \mathrm{ml} \mathrm{x} \mathrm{kg} \mathrm{x} \mathrm{hr.,} \mathrm{cantidad} \mathrm{significa-}$ tivamente menor que en el grupo de TRO.

Sólo un paciente del grupo de TRO tenja hiponatremia al ingresar, normalizándose su concentración de sodio plasmático a las seis horas de tratamiento; en el control de las seis horas dos niños que habian ingresado con natremia normal estaban hiponatrémicos. A las 24 horas en todos los pacientes se había normalizado el sodio plasmático. No se observaron casos de hipernatremia.

De los diez casos del grupo testigo, uno tenía hiponatremia al ingresar, dos a las 6 horas y uno a las 14 horas, no diferenciándose del grupo de TRO ел este aspecto (Tabla 3 ).

Tabla 3,

Hiponatremia al Ingreso y a las 6 y 24 horas de Tratamiento en los Grupos con Terapia Oral (TRO) y Parenteral (Control)

HIPONATREMIA

\begin{tabular}{lcccc} 
& Ingreso & 6 horas & 24 horas & Total \\
\hline $\begin{array}{l}\text { TRO } \\
\text { (27 casos) }\end{array}$ & 1 & 2 & 0 & 3 \\
$\begin{array}{l}\text { Control } \\
\text { (10 casos) }\end{array}$ & 1 & 2 & 1 & 4 \\
\hline Total & 2 & 4 & 1 & 7 \\
\hline
\end{tabular}

La magnitud de la deshidratación al ingreso, calculada según la proporción de recuperación del peso al término de la hidratación, era modera- 
da en cinco pacientes del grupo de TRO y en uno del grupo control, correspondiendo el resto de los casos a deshidrataciones leves.

No se encontró asociación entre resultado de la TRO, el sexo, el estado nutricional y el grado de deshidratación en el ingreso.

El volumen promedio de ingesta en las primeras seis horas de TRO fue $79.5 \mathrm{ml} \times \mathrm{kg}$, en los pacientes tratados con éxito y de $60 \mathrm{ml} \mathrm{x} \mathrm{kg}$. en los fracasos, diferencia estadísticamente significativa.

No hubo diferencia en los días de estada entre los pacientes con TRO y el grupo testigo tratado con terapia parenteral.

No se aislaron bacterias patógenas en las deposiciones en ninguno de los pacientes de ambos grupos; no se efectuaron estudios virológicos.

\section{COMENTARIO}

Esta experiencia confirma el buen resultado de la terapia rehidratante oral para la deshidratación secundaria a diarrea aguda demostrado en gran número de publicaciones previas.

No nos fue posible encontrar variables que se asociaran con el fracaso del tratamiento, pues los cuatro casos en que esto ocurrió no eran diferentes al resto en cuanto a edad, sexo, estado nutricional ni pérdidas por diarrea o vómitos. Esta falta de asociación puede deberse al reducido número de casos con mala respuesta; tal vez en series mayores, con un número proporcionalmente más alto de fracasos, pudieran encontrarse asociaciones que ayudaran a predecir en qué pacientes el tratamiento oral no dará buen resultado.

Sólo en uno de los cuatro fracasos detectamos pérdidas por deposiciones mayores de $10 \mathrm{ml} \mathrm{x}$ kg. $x$ hr., valor que se ha postulado como indicador de alta probabilidad de fracaso ${ }^{5}$; este hecho, agregado a la menor ingesta de líquido en los fracasos sugiere que en ellos la rehidratación fue más lenta y que pudiera haberse logrado suministrando TRO durante un tiempo mayor que las seis horas definidas por el estudio o si se hubiera empleado gastroclisis para aportar liquidos en lugar de la técnica de oferta frecuente.

La terapia parenteral no tendria ventajas sobre la vía oral en el tratamiento de la deshidratación leve y moderada debida a diarrea aguda, existien. do en cambio una serie de ventajas potenciales de la TRO en cuanto a costos, infecciones nosocomiales, pétdida de peso asociado al episodio diarreico, confort para el paciente, etc.

En nuestra opinión la TRO debe considerarse tratamiento de elección de la deshidratación debida a diarrea aguda, reservando el tratamiento endovenoso para los casos de deshidratación gra. ve.

\section{RESUMEN}

Se compara la terapia rehidratante oral (TRO) con el tratamiento parenteral en dos grupos de lactantes con diarrea aguda y deshidratación leve o moderada. A las seis horas de tratamiento se logró hidratar a 23 de los 27 pacientes del grupo TRO y a 5 de los 10 pacientes con terapia parenteral, diferencia estadisticamente significativa. No se encontraron difetencias en los valores de sodio plasmático entre ambos grupos; ni se observaron casos de hipernatremia. Los casos tratados con buen éxito por vía oral presentaron ingestas de liquidos significativamente mayores, los fracasos (79 vs. $60 \mathrm{ml} \mathrm{x} \mathrm{kg.} \mathrm{x} 6$ horas), no diferenciándose por las pérdidas en vómitos y deposiciones.

Se concluye que la TRO es un método efectivo para el tratamiento de lactantes con diarrea aguda y deshidratación, tal como ha sido amplia . mente demostrado en publicaciones previas.

\section{REFERENCIAS}

1. Finberg L.: Oral rehyduation for diatrhea, J. Pediatr. 101: 497, 1982.

2. Organización mundial de lo Salud: Terapia de rehiduatación oral: una bibliografía anotada, Segunda edición, Organización Panamericana de la Salud, Washington D.C. 1983.

3. Cleory T.G. et al: Relationship of oral rehydration solution to hypernatremia in infantile diarrhea, $J$. Pediats. 99:739, 1981 .

4. Nalin D.R. et al: Comparison of low and high sodium and potasium content in oral rehydration solutions, J, Pediatr. 97: 848, 1980.

5. Santosham $\boldsymbol{M}$. ef at: Oral rehydration therapy of infantile diarrhea, N. Engl, J. Med. 306: 1070 , 1982.

6. Walker $S$.: Dangers of Hydra-Lyte, Pediatrics 68 463, 1981.

7. Santoshen $M$. et al: Oral electrolyte solutions for infantile disrrhea, N. Engl. J. Med. 305: 581, 1981.

8. Pizarro D. ef al: Treatment of neonates with diarrhea with oral glucose-electrolytes solutions, J. Pediatr. 102: 153, 1983.

9. Gonzilez $R$, et al: Hidratación por vía oral. Experiencia en 100 lactantes deshidratados por diarrea, Pediatría 23: 178, 1980 .

10. Robson A.: General considerations in the care of sick children. En: Nelson Textbook of Pediatrics, 12th edition, 1983, W.B. Saunders Company, Philadelphia, pag. 233.

11. Duffau G. et al: Evaluación de dos fórmulas para hidratación en el síndrome diarreico agudo del lactante, Bol. Méd. Hosp. Infant. Méx. 39: 729, 1982.

12. Pizarro D. et at Hypernatremic diarcheal dehydration treated with "slow" oral rehydration therapy: a preliminary report, J. Pediatr. 104: 316, 1984. 